\title{
Image-guided stereotactic radiotherapy of the resection cavity for single resectable brain metastases
}

\author{
Harun Badakhshi ${ }^{1 *}$, Harald Lux ${ }^{1}$, David Kaul', Martin Misch² and Volker Budach ${ }^{1}$ \\ *Correspondence: Harun.Badakhshi@charite.de \\ CrossMark \\ $\leftarrow$ Click for updates \\ 'Department of Radiation Oncology, Charité School of Medicine and University Hospital Berlin, Germany. \\ ${ }^{2}$ Department of Neurosurgery, Charité School of Medicine and University Hospital Berlin, Germany.
}

\begin{abstract}
Objectives: To determine if the postoperative delivery of fractionated stereotactic radiotherapy (FSRT) for resection cavity for patients with single and resectable brain metastases is safe and effective.

Methods: A prospective feasibility protocol was set up to include patients with single and resectable brain metastases who underwent surgery and had low risk profile according to RPA classification. Fractionated stereotactic radiotherapy was applied. Single dose: $3.8 \mathrm{~Gy}$, total dose: 41.8 or $49.6 \mathrm{~Gy}$.

Results: There was no case of break due to clinical problems. There was no case of delay of FSRT. The onset acute toxicity was observed in 40 cases (76.9\%), no grade 3 and more was seen. Local recurrence free survival was 32.6 months, local control rate at $6,12,18$ and 24 months were $85 \%, 77.9 \%, 65.9 \%$ and $65.9 \%$. Overall local failure occurred in $34.1 \%$ of patients. Overall survival rates at $6,12,18$ and 24 months were $90.3 \%, 63.9 \%, 47.7 \%$ and $31.6 \%$. Median survival was 18.3 months (13.8-22.8) and overall $17.3 \%$ were living at the time of last analysis. Distant control rates at $6,12,18$ and 24 months were $49.4 \%, 38.2 \%, 25.5 \%$ and $22.3 \%$. Median distant recurrence free survival was 6 months (0-12.0) with overall distant failure in $77.7 \%$ of patients.

Conclusion: FSRT for surgical cavity might be one possible option in treatment of single and resectable brain metastases.
\end{abstract}

Keywords: Brain metastases, stereotactic radiosurgery, image-guided intervention, local control

\section{Introduction}

Brain metastases occur in approximately $30 \%$ of patients with metastasized cancer, greatly shortening their life expectancies as well as detrimentally effecting personal and societal quality of life [1]. Prognostic assessments using validated scoring systems can help clinicians select approaches and management strategies for brain metastases that are tailored to the individual characteristics of the patient and his or her disease. Relevant scoring systems have been developed based on recursive partitioning analysis (RPA) and graded prognostic assessments (GPA) [2].

The exact management of patients with favorable or intermediate prognoses (RPA I and II, respectively; GPA scores $\geq 3$ and 1.5-2.5) can vary depending on the number of metastases [3]. However, whole-brain radiotherapy (WBRT) or, in the case of resectable tumors, surgery has traditionally been performed for patients with these favorable and intermediate prognoses. Level I evidence indicates that the combination of WBRT and surgery for resectable single metastases is better than surgery alone in terms of local control (LC; $46 \%$ versus 10\%), but does not confer any survival benefits [4]. Because the addition of WBRT has not been observed to improve survival, physicians and patients may be hesitant to combine WBRT with surgery.
Indeed, there are some disadvantages to WBRT besides its lack survival benefit. First, WBRT has several acute side effects, including hair loss, skin irritation, nausea, vomiting, and fatigue. Second, WBRT can have a clinically and statistically significant impact on neurocognitive function, thereby reducing patients' quality of life after treatment $[\mathbf{5 , 6 ]}$. Third, WBRT standard doses of $30 \mathrm{~Gy}$ (in 10 fractions) and $20 \mathrm{~Gy}$ (in 4 or 5 fractions) appear to be suboptimal for cancers that are relatively resistant to radiotherapy, such as melanoma and renal cell carcinoma [7]. Because of these disadvantages, there is a recognized need for alternative treatment strategies. At present, WBRT is vastly used in cases that have especially favorable characteristics: a single resectable cerebral lesion with a good KPS, patient age $<65$ years, and a controlled or potentially controllable primary tumor. It may be considered in these cases as overtreatment. A variety of alternative therapeutic options is available in these favorable clinical scenarios, including focal high-precision radiotherapy, which we decided to investigate. However, because of the experimental nature of focal high-precision radiotherapy, we decided to begin our investigation more cautiously, using fractionated stereotactic radiotherapy (FSRT), which is a somewhat less aggressive technique.

Little data are available on hypofractionated stereotactic 
Badakhshi et al. Medical Imaging and Radiology 2014,

http://www.hoajonline.com/journals/pdf/2054-1945-2-3.pdf

doi: $10.7243 / 2054-1945-2-3$

radiation therapy with a single daily dose $>2.2$ Gy up to a total dose of 40-50 Gy that is delivered to the resection cavity after removal of a single and resectable brain metastasis. We specifically designed our study to examine the feasibility and safety of image-guided FSRT, sought to determine whether FSRT was an effective option for single and resectable brain metastases. In the present study, we describe our clinical experiences focusing on issues of reproducibility, patient safety and clinical outcome.

\section{Material and methods}

\section{Patient selection and study endpoints}

Demographic data for all patients were obtained from a prospectively maintained database. Informed consent was obtained from all patients before treatment. The local review committee approved the protocol. We identified 61 patients who had single brain metastases (which had resulted from a variety of different cancers), all of whom underwent resection followed by FSRT between January 2010 and December 2012. Of the 61 identified cases, 52 met our protocol's inclusion criteria: a single resectable cerebral lesion with KPS $>60$ and a controlled or potentially controllable primary tumor. We did not exclude patients older than 65 years, so long as they were physically fit, active, younger than 75 years, but explicitly wished to receive FSRT.

The median age of included patients was 62.8 years (31-74 years). The study cohort comprised 30 female and 22 male patients. One experienced team performed the surgical procedures, and a second experienced team performed the radiotherapeutic procedures. A multidisciplinary team made clinical decisions. The primary endpoints of the study were technical and medical feasibility, including workflows and FSRT-related toxicity greater than grade 2 , according to the Common Terminology Criteria for Adverse Events (CTC AE, version 4.0). The secondary endpoint was local tumor control within the irradiated region.

\section{Treatment}

All patients were treated with image-guided frameless (linac)based FSRT. The majority of patients $(n=38,73 \%)$ received $41.8 \mathrm{~Gy}$ at 11 fractions of $3.8 \mathrm{~Gy}$. The remaining patients ( $\mathrm{n}=14$, $26.9 \%$ ) received two additional fractions, increasing the total to 49.4 Gy (13 fractions of 3.8 Gy).

Patient immobilization was achieved using a commercially available head mask fixation system (Brainlab AG, Feldkirchen, Germany). The specific characteristics of the system and principles of the technique have been described previously [8]. The clinical target volume (CTV) was identified on the basis of $1 \mathrm{~mm}$ gadolinium-enhanced axial MRI (Siemens Medical Systems, Erlangen, Germany) images fused with computed tomography (Siemens Medical Systems, Erlangen, Germany) image data sets. Scanning was conducted in the spiral mode using a pitch of 0.75 , and slices of $1.2 \mathrm{~mm}$ thickness and spacing were acquired for the entire cranium. Surrounding edema or the operative corridor was not included. The dose prescription was made to the planning target volume (PTV), which consisted of the CTV with an added $2 \mathrm{~mm}$ margin around it as the planning target volume (PTV). To ensure that at least $95 \%$ of the PTV received the prescribed dose, doses were prescribed constantly to the $80 \%$ isodose line, as normalized to the maximum dose. Treatment volumes were achieved with 3-5 dynamic arcs using a dedicated Novalis accelerator (Brainlab AG, Feldkirchen, Germany). Dexamethasone therapy was started by the first day of treatment, orally administered at doses of 4-6 mg per day, and maintained for 10 days following the end of treatment.

\section{Follow-up}

Patients were clinically observed before and during FSRT. The first follow-up appointment was scheduled three months later, with additional appointments occurring at least once every six months. We planned to document treatment-related toxicity in first three months following FSRT. Local progression was defined as new contrast enhancement in the PTV. Distant intracranial progression was defined by the presence of new brain metastases or leptomeningeal enhancement outside the PTV. For each patient who died, we attempted to determine the cause of death.

\section{Statistics}

LC, distant control (DC), and OS were defined as beginning at the time of FSRT and were estimated using the KaplanMeier method. In univariate analyses, we used the log-rank test to assess survival differences for categorical variables and the Cox proportional hazards model to assess survival differences for continuous variables. Significant prognostic factors $(p<0.05)$ were included in a multivariate analysis, using a Cox proportional hazards regression model. Statistical evaluations were performed using a commercial statistical software package (SPSS version 20.0, IBM Germany GmBH).

\section{Results}

Patient and tumor characteristics are summarized in Table 1. The most common primary tumors were non-small cell lung cancer $(16 / 52,30.8 \%)$, breast cancer $(10 / 52,21.1 \%)$, melanoma $(7 / 52,9.2 \%)$, and colorectal cancer (5/52, 6.6\%). Median follow-up time with imaging was 9.6 months (1-45 months).

The median interval between surgery and FSRT was 1.9 months (0.53-4.01 months). Median KPS at the time of FSRT was 80 (60-90). Ten patients were excluded from the analyses of LC, DC, and salvage therapy because sufficient imaging data were unavailable.

\section{Primary endpoints: feasibility, acute toxicity}

With respect to feasibility, we observed that all patients completed FSRT. No cases involved breaks or pauses in FSRT because of adverse responses. In three cases, there was a short break (1-2 days) because of technical problems with 
Badakhshi et al. Medical Imaging and Radiology 2014,

Table 1. Patient characteristics, $n=52$.

\begin{tabular}{|c|c|}
\hline \multicolumn{2}{|l|}{ Age (years) } \\
\hline & median $62.8(31-75)$ \\
\hline \multicolumn{2}{|l|}{ Gender } \\
\hline & $\mathrm{m}: 22(42.3 \%)$ \\
\hline & w: $30(57.7 \%)$ \\
\hline \multicolumn{2}{|c|}{ KPS median (\%) } \\
\hline & median 80 (range: $60-90$ ) \\
\hline & 60: $11(21.1 \%)$ \\
\hline & 70: $13(25 \%)$ \\
\hline & 80: $15(28.8 \%)$ \\
\hline & 90: $13(25 \%)$ \\
\hline \multicolumn{2}{|c|}{ RPA classification } \\
\hline & 1: $18(35 \%)$ \\
\hline & 2: $25(48 \%)$ \\
\hline & $3: 9(17 \%)$ \\
\hline \multicolumn{2}{|l|}{ FSRT doses } \\
\hline & 41.8 Gy: $3873.03 \%$ \\
\hline & $49.6 \mathrm{~Gy}: 1426.9 \%$ \\
\hline \multicolumn{2}{|c|}{ Primary cancer } \\
\hline & Lung: $16(30.8 \%)$ \\
\hline & Breast: $10(19.2 \%)$ \\
\hline & Melanoma: 7 (13.5\%) \\
\hline & Colorectal: 5 (9.6\%) \\
\hline & CUP: 3 (5.8\%) \\
\hline & Other \\
\hline \multicolumn{2}{|c|}{ Localization of brain metastasis } \\
\hline & Cerebellum 13 (25\%) \\
\hline & Frontal $12(23.1 \%)$ \\
\hline & Parietal $9(17.3 \%)$ \\
\hline & Temporal 5 (9.6\%) \\
\hline & Occipital $5(9.6 \%)$ \\
\hline & Other \\
\hline
\end{tabular}

the machines. All delays in the time of FSRT were scheduled in advance; there were no unscheduled delays.

With respect to FSRT-related toxicity, new-onset acute toxicity was observed in 40 cases (76.9\%). However, none of these cases involved acute toxicity of grade 3 or higher. Patients reported manageable fatigue (grade $2, n=32,61.5 \%$ ), lethargy (grade $2, n=32,61.5 \%$ ), headache (grade $2, n=18$, $34.6 \%$ ), dizziness, (grade $2, n=12,23 \%$ ), and ataxia (grade $2, n=6,11.5 \%)$. FSRT-related toxicity is presented in Figure 1.

\section{Secondary endpoint: local control}

In 10 cases a recurrence occurred. Median local recurrence-free survival was 32.6 months. LC rates at $6,12,18$, and 24 months were $85 \%, 77.9 \%, 65.9 \%$, and $65.9 \%$, respectively (Figure 1). Overall, local failure occurred in $34.1 \%$ of patients. Univariate analysis identified residual tumor mass after resection as a highly significant factor associated with local failure $(p<0.001)$.

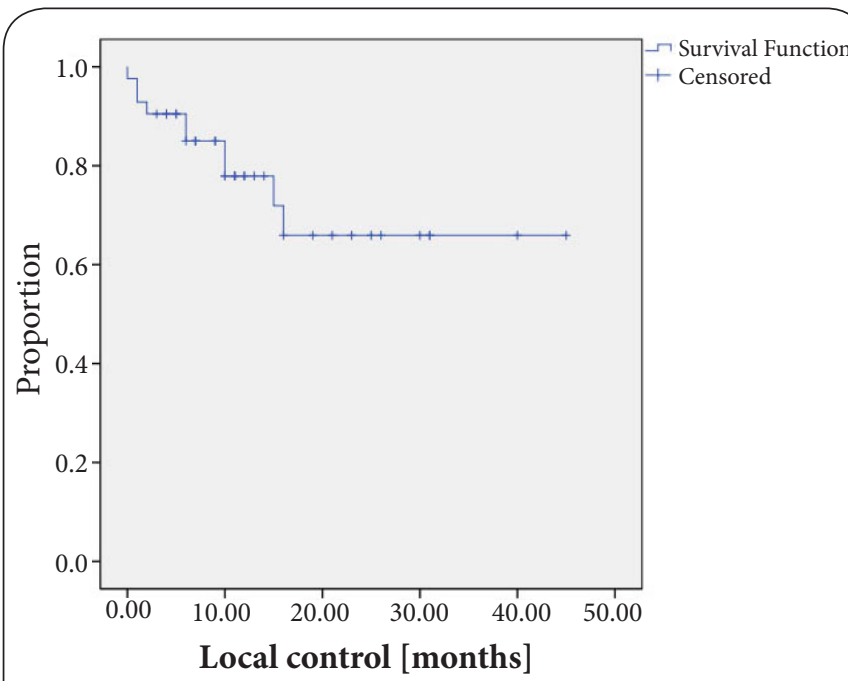

Figure 1. Local in-field control for resection cavity irradiation in single brain metastases.

Tertiary endpoint: overall survival and distant control Median survival was 18.3 months (range: 13.8-22.8 months), with $17.3 \%$ of patients alive at the time of last follow-up. OS rates at $6,12,18$, and 24 months were $90.3 \%, 63.9 \%, 47.7 \%$, and $31.6 \%$ (Figure 2). RPA and KPS were identified prognostic factors for OS. Univariate analysis also indicated that RPA class 2 was predictive of OS $(p<0.02)$, and that RPA class 1 was associated with better survival than RPA class $3(p<0.061)$. An analysis of patients stratified according to KPS (Group $1 \mathrm{KPS}$ : 80-90, Group 2 KPS: 60-70) confirmed KPS of Group 1 was predictive of better survival $(p<0.048)$.

DC rates at $6,12,18$, and 24 months were $49.4 \%, 38.2 \%$, $25.5 \%$, and $22.3 \%$, respectively. Median distant recurrence-free

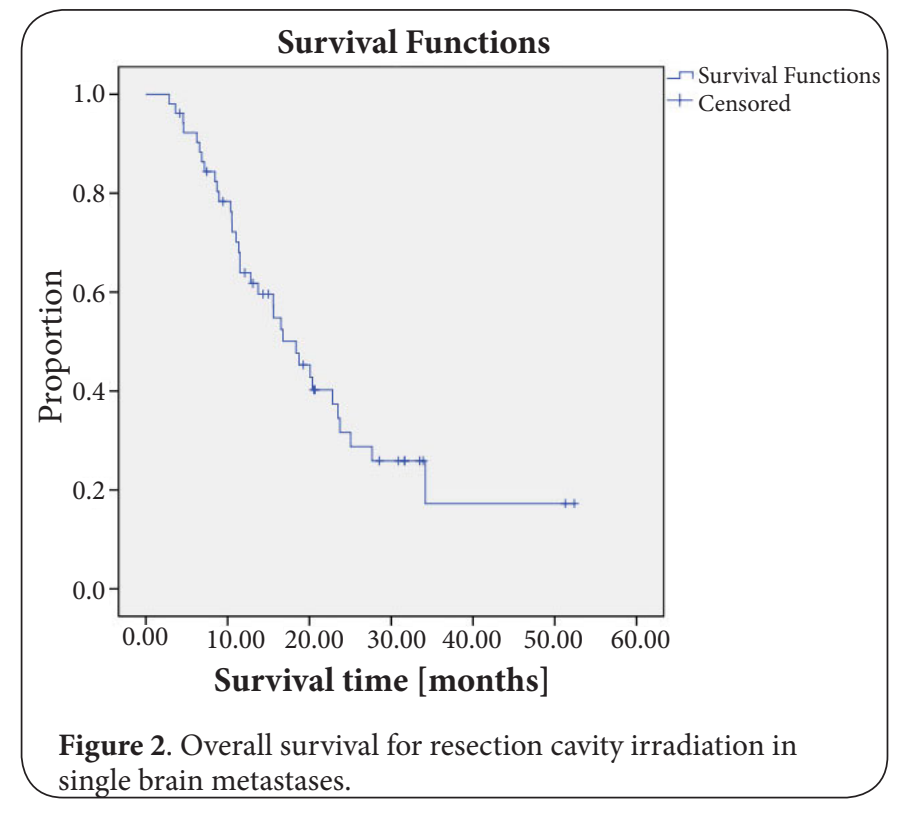


Badakhshi et al. Medical Imaging and Radiology 2014,

http://www.hoajonline.com/journals/pdf/2054-1945-2-3.pdf

doi: $10.7243 / 2054-1945-2-3$

survival was 6 months (range: $0-12.0$ months) with distant failure ultimately occurring in $77.7 \%$ of patients.

\section{Salvage therapy}

Twenty-six of 42 patients (61.9\%) received salvage therapy: $42.3 \%$ received WBRT $(n=11), 42.3 \%$ received FSRT $(n=11)$, and $9.5 \%$ underwent resection $(n=4)$. Among the 26 patients, the median time to salvage therapy was 5.3 months (range: 1.87-19.91 months). The median subsequent survival time was 8.4 months (range: $5.2-11.6$ months). In $69.23 \%$ of cases $(18 / 26)$, the patient received initial salvage therapy because of distant failure alone.

\section{Discussion}

Our results demonstrate the technical and medical feasibility of focal high-precision radiotherapy (FSRT) of the resection cavity after removal of single brain metastases. Our decision to defer investigation of whole-brain radiotherapy was motivated by the only prior study that provided level 1 evidence [4] and the few other relevant studies that provided lower-level evidence and were available when we planned our study [9-12]. We were additionally motivated by outcome data showing that, for a variety of cancers, improvements in systemic therapy lead to longer survival times.

Currently, the delayed effects of WBRT are becoming a more serious issue. During study planning, we generated a specific hypothesis for investigation: WBRT is deferrable, and it is better to postpone WBRT for patients who have resectable single brain metastases and favorable risk profiles, and therefore longer life expectancies. However, WBRT undoubtedly reduces local and regional recurrence when assessed in a prospective setting $[4,13]$. For patients with multiple, unresectable or medium-high risk brain metastases, WBRT should remain the standard treatment.

\section{Technical and medical feasibility}

Our preliminary results indicate that FSRT is feasible from a technical perspective, at least for departments that have sufficient technical infrastructure and experienced teams. FSRT is also safe, causing no severe side effects. However, the duration of FSRT may be a critical limitation; FSRT is delivered over the course of two weeks. To date, few studies have considered hypofractionation. To our knowledge, this study's dose regimen (a single dose of 3.8 Gy delivered in $11-13$ fractions) is the only regimen of this type.

Few previous studies of FSRT have reported toxicity. In a recent study of 33 cases, Steinmann et al., reported no toxicities of grade 2 or higher [14]. Their study examined a dose prescription that nominally differed from our own, but was actually similar in terms of biologic equivalence. The authors reported side effects including mild alopecia, dermatitis, and fatigue. Only eight patients received corticosteroids at the end of radiotherapy. In an excellent study of 33 patients with solitary brain metastases, Connolly et al., reported side effects experienced during and after a hypofractionated radiotherapy regimen ( $2.67 \mathrm{~Gy}, 15$ times) that was similar to our own. It was observed that none of the patients experienced additional neurological symptoms related to the therapy [15].

\section{Local control}

A $65.9 \% \mathrm{LC}$ rate was observed in the present study. This is the lowest LC rate of among the studies compared, possibly because $9.5 \%$ of patients included in the estimation of intracranial control had residual tumor mass, which was strongly associated with local failure. We observed no significant difference in LC rates between the two dose regimens. The median prescription dose of $41.8 \mathrm{~Gy}$ was comparable to studies with a wide range of $\mathrm{LC}$ rates. For example, in a study with a prescription dose of $40 \mathrm{~Gy}$, Steinmann et al., observed a $73 \%$ LC rate [14], while in a study with a prescription dose of $40.5 \mathrm{~Gy}$, Connolly et al., observed an $85 \%$ LC rate [15]. Considering the small sizes of study cohorts, the LC rate that was observed in the present study may be not be unusual at all (Table 2).

Table 2. Relevant studies.

\begin{tabular}{llllll}
\hline Study & Patients & Lesions & $\begin{array}{l}\text { Local } \\
\text { failure }\end{array}$ & $\begin{array}{l}\text { Regional } \\
\text { failure }\end{array}$ & Survival \\
\hline$[\mathbf{1 6}]$ & 56 & 1 & $8.90 \%$ & $37.50 \%$ & 20.5 \\
{$[\mathbf{1 4}]$} & 33 & 1 & $27.00 \%$ & $47.00 \%$ & 20 \\
{$[\mathbf{1 5}]$} & 33 & 1 & $15.00 \%$ & $39.00 \%$ & 30.7 \\
Present study & 52 & 1 & $34.10 \%$ & $77.70 \%$ & 18.3 \\
\hline
\end{tabular}

\section{Overall survival}

Median OS times have ranged from 18.3 to 30.7 months in studies of patients with single brain metastasis who received focal radiotherapy after resection [14-16], including the OS in the present study. In comparison, median OS times have ranged from 11 to 17 months for cohorts that include multiple brain metastases [10-12,17-22].

Among patients with brain metastases, single brain metastasis is predictive of longer survival [11]. Accordingly, the present study's median survival time of 18.3 months reasonably consistent with previous reports, particularly in comparison to the approximately 20 month median OS that was observed in a prior study of a similar number of patients [16]. Three retrospective studies of single brain metastases that incorporated the present study's treatment mode reported a median survival time of 20-30.7 months [14-16], whereas studies with initial postoperative WBRT reported median survival times of 10.7-12 months [4,13]. Therefore, research on the difference in survival times for patients with single brain metastasis could provide a major argument for proper treatment.

Our analysis of RPA indicated that classes 1 and 2 were associated with better OS than class 3 . However, we did 
Badakhshi et al. Medical Imaging and Radiology 2014,

not observe any significant difference in OS between RPA classes 1 and 2, perhaps because the factors contributing to RPA classification (age, extra-cranial metastases) were not significantly associated with OS in our univariate analyses. Therefore, KPS could be the major factor that contributes to the survival differences that were observed. Additionally, the number of patients and the case censoring varied across RPA classes.

\section{Distant intracranial control}

The 6-month median DC time fell short of the18.3-month median OS time. The rather low $22.3 \%$ DC rate suggests the underlying reason for the majority of first salvage interventions (69.2\% of salvage therapies followed loss of DC). Therefore, patients were burdened by complications of distant metastases and the associated therapeutic aftermath. Higher DC rates have previously been reported for WBRT as standard treatment after surgery (range: 76-86\%) [4,13].

These findings lead us to question the value of enhanced DC conferred by WBRT, drawing attention to the specifics of the application of WBRT in the present study. First, STs of the relevant cases were conducted after a median of 5.37 months. In the present study, $26.19 \%$ of first salvage therapies after recurrence were WBRT. Compared with traditional upfront WBRT, use of WBRT as salvage therapy delays possible side effects. Second, $16.7 \%(7 / 42)$ of people in the present study lived longer than 18 months without recurrence, and 11.9\% (5/42) lived longer than two years without recurrence. These patients are proof that WBRT is not necessary for long-term survival. Indeed, these patients entirely avoided the risks of upfront WBRT. Apart from these two arguments in favor of image-guided stereotactic radiotherapy, there is little evidence concerning the balance of benefits and harms of upfront WBRT and WBRT as a salvage therapy after DF.

However, despite the side effects of WBRT $[5,23]$, which include loss of health-related quality of life [24], there is some contradictory evidence concerning its effects. Indeed, overall neurocognitive benefit has been claimed [25], as well as the possibility of reducing harm by sparing portions of the brain $[26,27]$.

\section{Conclusion}

In the last decade, the developments of innovative technologies have made focal radiotherapy a viable, safe, and effective option of treatment. Accordingly, the predominance of WBRT treatment strategies should be critically revised. The use of WBRT as a one-size-fits-all treatment should be reconsidered. In the present study, we examined FSRT of the surgical cavity and demonstrated that it could be a treatment option for single and resectable brain metastases.

\section{Competing interests}

The authors declare that they have no competing interests.

Authors' contributions
\begin{tabular}{|l|c|c|c|c|c|}
\hline Authors' contributions & HB & HL & DK & MM & VB \\
\hline Research concept and design & $\checkmark$ & $\checkmark$ & $\checkmark$ & $\checkmark$ & $\checkmark$ \\
\hline Collection and/or assembly of data & $\checkmark$ & $\checkmark$ & -- & -- & -- \\
\hline Data analysis and interpretation & $\checkmark$ & $\checkmark$ & -- & -- & -- \\
\hline Writing the article & $\checkmark$ & $\checkmark$ & -- & -- & -- \\
\hline Critical revision of the article & $\checkmark$ & $\checkmark$ & $\checkmark$ & $\checkmark$ & $\checkmark$ \\
\hline Final approval of article & $\checkmark$ & $\checkmark$ & $\checkmark$ & $\checkmark$ & $\checkmark$ \\
\hline Statistical analysis & $\checkmark$ & $\checkmark$ & -- & -- & - \\
\hline
\end{tabular}

\section{Acknowledgement}

We thank to all doctors and nurses of both departments involved in treatment of our patients.

\section{Publication history}

Editor: Maythem Saeed, University of California, San Francisco, USA. Received: 31-Oct-2013 Final Revised: 26-Mar-2014

Accepted: 16-Apr-2014 Published: 26-Apr-2014

\section{References}

1. Nieder C, Spanne O, Mehta MP, Grosu AL and Geinitz H. Presentation, patterns of care, and survival in patients with brain metastases: what has changed in the last 20 years? Cancer. 2011; 117:2505-12. | Article PubMed

2. Sperduto PW, Berkey B, Gaspar LE, Mehta M and Curran W. A new prognostic index and comparison to three other indices for patients with brain metastases: an analysis of 1,960 patients in the RTOG database. Int J Radiat Oncol Biol Phys. 2008; 70:510-4. | Article | PubMed

3. Gondi V and Mehta MP. Novel insights into the management of brain metastases. Curr Opin Neurol. 2010; 23:556-62. | Article | PubMed

4. Patchell RA, Tibbs PA, Regine WF, Dempsey RJ, Mohiuddin M, Kryscio RJ, Markesbery WR, Foon KA and Young B. Postoperative radiotherapy in the treatment of single metastases to the brain: a randomized trial. JAMA. 1998; 280:1485-9. | Article I PubMed

5. Chang EL, Wefel JS, Hess KR, Allen PK, Lang FF, Kornguth DG, Arbuckle RB, Swint JM, Shiu AS, Maor MH and Meyers CA. Neurocognition in patients with brain metastases treated with radiosurgery or radiosurgery plus whole-brain irradiation: a randomised controlled trial. Lancet Oncol. 2009; 10:1037-44. | Article | PubMed

6. Welzel G, Fleckenstein K, Schaefer J, Hermann B, Kraus-Tiefenbacher $U$, Mai SK and Wenz F. Memory function before and after whole brain radiotherapy in patients with and without brain metastases. Int J Radiat Oncol Biol Phys. 2008; 72:1311-8. | Article | PubMed

7. Wronski M, Maor MH, Davis BJ, Sawaya R and Levin VA. External radiation of brain metastases from renal carcinoma: a retrospective study of 119 patients from the M. D. Anderson Cancer Center. Int J Radiat Oncol Biol Phys. 1997; 37:753-9. I Article I PubMed

8. Wurm RE, Erbel S, Schwenkert I, Gum F, Agaoglu D, Schild R, Schlenger $L$, Scheffler D, Brock M and Budach V. Novalis frameless image-guided noninvasive radiosurgery: initial experience. Neurosurgery. 2008; 62:A11-7. | Article | PubMed

9. Soltys SG, Adler JR, Lipani JD, Jackson PS, Choi CY, Puataweepong P, White $\mathrm{S}$, Gibbs IC and Chang SD. Stereotactic radiosurgery of the postoperative resection cavity for brain metastases. Int J Radiat Oncol Biol Phys. 2008; 70:187-93. | Article | PubMed

10. Mathieu D, Kondziolka D, Flickinger JC, Fortin D, Kenny B, Michaud K, Mongia S, Niranjan A and Lunsford LD. Tumor bed radiosurgery after resection of cerebral metastases. Neurosurgery. 2008; 62:817-23. | Article | PubMed

11. Karlovits BJ, Quigley MR, Karlovits SM, Miller L, Johnson M, Gayou O and Fuhrer R. Stereotactic radiosurgery boost to the resection bed for 
Badakhshi et al. Medical Imaging and Radiology 2014,

oligometastatic brain disease: challenging the tradition of adjuvant whole-brain radiotherapy. Neurosurg Focus. 2009; 27:E7. | Article | PubMed

12. Do L, Pezner R, Radany E, Liu A, Staud C and Badie B. Resection followed by stereotactic radiosurgery to resection cavity for intracranial metastases. Int J Radiat Oncol Biol Phys. 2009; 73:486-91. | Article | PubMed

13. Kocher M, Soffietti R, Abacioglu U, Villa S, Fauchon F, Baumert BG, Fariselli L, Tzuk-Shina T, Kortmann RD, Carrie C, Ben Hassel M, Kouri $M$, Valeinis $E$, van den Berge $D$, Collette $S$, Collette $L$ and Mueller RP. Adjuvant whole-brain radiotherapy versus observation after radiosurgery or surgical resection of one to three cerebral metastases: results of the EORTC 22952-26001 study. J Clin Oncol. 2011; 29:134-41. | Article | PubMed Abstract | PubMed Full Text

14. Steinmann D, Maertens B, Janssen S, Werner M, Fruhauf J, Nakamura $M$, Christiansen $\mathrm{H}$ and Bremer M. Hypofractionated stereotactic radiotherapy (hfSRT) after tumour resection of a single brain metastasis: report of a single-centre individualized treatment approach J Cancer Res Clin Oncol. 2012; 138:1523-9. | Article | PubMed

15. Connolly EP, Mathew M, Tam M, King JV, Kunnakkat SD, Parker EC, Golfinos JG, Gruber ML and Narayana A. Involved field radiation therapy after surgical resection of solitary brain metastases--mature results. Neuro Oncol. 2013; 15:589-94. | Article | PubMed

16. Ogiwara H, Kalakota K, Rakhra SS, Helenowski IB, Marymont MH, Kalapurakal JA, Mehta MP, Levy RB and Chandler JP. Intracranial relapse rates and patterns, and survival trends following post-resection cavity radiosurgery for patients with single intracranial metastases. $J$ Neurooncol. 2012; 108:141-6. | Article | PubMed

17. Rwigema JC, Wegner RE, Mintz AH, Paravati AJ, Burton SA, Ozhasoglu C and Heron DE. Stereotactic radiosurgery to the resection cavity of brain metastases: a retrospective analysis and literature review. Stereotact Funct Neurosurg. 2011; 89:329-37. | Article | PubMed

18. Choi CY, Chang SD, Gibbs IC, Adler JR, Harsh GRt, Lieberson RE and Soltys SG. Stereotactic radiosurgery of the postoperative resection cavity for brain metastases: prospective evaluation of target margin on tumor control. Int J Radiat Oncol Biol Phys. 2012; 84:336-42. | Article | PubMed

19. Soltys SG, Adler JR, Lipani JD, Jackson PS, Choi CY, Puataweepong P, White $S$, Gibbs IC and Chang SD. Stereotactic radiosurgery of the postoperative resection cavity for brain metastases. Int J Radiat Oncol Biol Phys. 2008; 70:187-93. | Article | PubMed

20. Jensen CA, Chan MD, McCoy TP, Bourland JD, deGuzman AF, Ellis TL, Ekstrand KE, McMullen KP, Munley MT, Shaw EG, Urbanic JJ and Tatter SB. Cavity-directed radiosurgery as adjuvant therapy after resection of a brain metastasis. J Neurosurg. 2011; 114:1585-91. | Article | PubMed Abstract | PubMed Full Text

21. Quigley MR, Fuhrer R, Karlovits S, Karlovits B and Johnson M. Single session stereotactic radiosurgery boost to the post-operative site in lieu of whole brain radiation in metastatic brain disease. J Neurooncol. 2008; 87:327-32. | Article | PubMed

22. Jagannathan J, Yen CP, Ray DK, Schlesinger D, Oskouian RJ, Pouratian $N$, Shaffrey ME, Larner J and Sheehan JP. Gamma Knife radiosurgery to the surgical cavity following resection of brain metastases. J Neurosurg. 2009; 111:431-8. | Article | PubMed

23. DeAngelis LM, Delattre JY and Posner JB. Radiation-induced dementia in patients cured of brain metastases. Neurology. 1989; 39:789-96. | Article | PubMed

24. Soffietti R, Kocher M, Abacioglu UM, Villa S, Fauchon F, Baumert BG, Fariselli L, Tzuk-Shina T, Kortmann RD, Carrie C, Ben Hassel M, Kouri $M$, Valeinis E, van den Berge D, Mueller RP, Tridello G, Collette $L$ and Bottomley A. A European Organisation for Research and Treatment of Cancer phase III trial of adjuvant whole-brain radiotherapy versus observation in patients with one to three brain metastases from solid tumors after surgical resection or radiosurgery: quality-of-life results. $J$ Clin Oncol. 2013; 31:65-72. | Article | PubMed

25. Li J, Bentzen SM, Renschler M and Mehta MP. Regression after wholebrain radiation therapy for brain metastases correlates with survival and improved neurocognitive function. J Clin Oncol. 2007; 25:1260-6. |
Article | PubMed

26. Gondi V, Tome WA and Mehta MP. Why avoid the hippocampus? A comprehensive review. Radiother Oncol. 2010; 97:370-6. | Article | PubMed Abstract | PubMed Full Text

27. Marsh JC, Gielda BT, Herskovic AM, Wendt JA and Turian JV. Sparing of the hippocampus and limbic circuit during whole brain radiation therapy: A dosimetric study using helical tomotherapy. J Med Imaging Radiat Oncol. 2010; 54:375-82. | Article | PubMed

\section{Citation:}

Badakhshi H, Lux H, Kaul D, Misch M and Budach $\mathrm{V}$. Image-guided stereotactic radiotherapy of the resection cavity for single resectable brain metastases. Med Imaging Radiol. 2014; 2:3. http://dx.doi.org/10.7243/2054-1945-2-3 